\title{
Oltre il canone nazionale. Le voci che parlano in catalano
}

\author{
Beyond the national fee. Voices speaking in Catalan
}

\author{
Pietro Cataldi \\ cataldi@unistrasi.it
}

Università per Stranieri di Siena

\begin{abstract}
Riassunto: Nella nuova Europa della generazione, o delle generazioni, Erasmus, in questa Europa che vogliamo cosmopolita, ci aspetta il racconto di una letteratura meno parziale, meno sottomessa al principio egemonico degli stati-nazione, una letteratura che ci permetta di recuperare, soprattutto, le voci forti ed emozionanti di autori che il fiume del processo storico ha lasciato in un'ansa laterale, dove piccole comunità hanno potuto continuare a coltivarne e proteggerne la memoria, non senza il rischio di partecipare, con la loro cura identitaria, al processo di esclusione dal canone internazionale. Potremo, anche, guardare per la prima volta, o per la prima volta con adeguata consapevolezza e rispetto, verso letterature emarginate e minorizzate come quella, in lingua catalana. Sappiamo quali faticose vicende storiche ne abbiano segnato la marginalizzazione, fino alla persecuzione franchista, ma proporrei di abbandonare questo punto di vista - che rischia di bloccarci in un gesto di rivendicazione ancora subalterna- a favore di una prospettiva che, se davvero vorrà essere inclusiva e accogliente, dovrà assumersi la responsabilità di essere capace di collocarsi nel presente.
\end{abstract}

Parole chiave: Letteratura catalana, studi del canone letterario, confini sovranazionali, ricezione

\begin{abstract}
In the new Europe of the generation, or generations, Erasmus, in this Europe that we want cosmopolitan, awaits us the story of a less partial literature, less subject to the hegemonic principle of nation-states, a literature that allows us to recover, above all, the strong and exciting voices of authors that the river of the historical process has left on one of its sides, where small communities have been able to continue to cultivate and protect their memory, not without the risk of participating, with their identity care, in the process of exclusion from the international canon. For the first time we also will be able to look with adequate awareness and respect at marginalized and minority literatures like the one in the Catalan language. We know what difficult historical events have marked its marginalization, up to the Franco persecution, but I would propose to abandon this point of view -which risks blocking us in a gesture of still subordinate claim- in favor of a perspective that, if it really wants to be inclusive and welcoming, will have to assume the responsibility of being able to place itself in the present.
\end{abstract}

Keywords: catalan literature, studies of the literary canon, supranational boundaries, reception 
1. I processi della globalizzazione hanno imposto un ripensamento dello spazio letterario; un ripensamento profondo, certo, ma forse non ancora abbastanza profondo. La separazione tra la letteratura insegnata nelle scuole e nelle università -soprattutto in un paese come l'Italia, da questo punto di vista radicato nelle sue tradizioni e inevitabilmente tradizionalista- e la letteratura concretamente praticata dai lettori reali al di fuori delle istituzioni formative si è accentuata fino a produrre, o quanto meno rischiare, un divorzio irreparabile: durante le ore di insegnamento a scuola, i docenti di letteratura discorrono per lo più di autori e di opere che non avranno mai un posto fra le loro libere letture, e di queste d'altra parte non dicono ai loro studenti, quasi si trattasse di un vizio colpevole. Parlano delle Prose della volgar lingua o degli Asolani di Pietro Bembo, della poesia di Monti e di Carducci, del teatro di Alfieri; ma a casa, nelle ore libere, leggono i grandi romanzi tradotti dall'inglese, dal francese, dal giapponese... E così si guardano dal coinvolgere i loro studenti nella letteratura che meglio potrebbe oggi interessare loro, e a partire dalla quale anche i grandi classici potrebbero forse acquistare di nuovo un senso e una ragione di interesse non coatto.

In Italia la condivisione del canone dei classici ha assolto alla funzione di rendere più forte e più coesa l'identità nazionale; e di questa Dante è stato, con Verdi, il collante più efficace e condiviso, così che tra i versi più belli della nostra letteratura un poeta sensibile alla questione delle identità, il triestino Umberto Saba, poteva indicare «la bocca mi baciò tutto tremante» del quinto dell'Inferno e «Udite tutti del mio cor gli affanni» dall'Ernani verdiano. Benché avessero meno bisogno dell'Italia, per ragioni storiche, di fondare questa identità sul codice letterario, gli inglesi si sono d'altra parte riconosciuti a lungo nel nome di Shakespeare; i francesi in quello di Corneille e di Victor Hugo, e magari nella Chanson de Roland; gli spagnoli nei capolavori del Siglo de Oro e magari nel Cantar de mio Cid; i tedeschi nella Weimar di Goethe. Sappiamo che oggi questa prospettiva è invecchiata: se non altro i processi della globalizzazione ne hanno fatto una forma di anacronismo politico e culturale; e le identità nazionali vengono riconosciute, nel grande spaventoso scambio mercantile che domina il pianeta, come brand vendibili ai quali affidare modelli e identità di tipo prevalentemente merceologico. L'Italia sarà dunque, prima ancora che il paese di Dante e di Verdi, quello della pizza e della Ferrari.

$\mathrm{Al}$ cospetto di questo processo di svuotamento, la rivendicazione del grande canone letterario nazionale, mentre crede di opporre un argine di civiltà alla deriva del tardo capitalismo, rischia in realtà di assumere $i$ tratti reazionari di un asfittico sovranismo. E per alcuni paesi europei, fra i quali l'Italia e la Spagna, anche la letteratura può diventare il modo per praticare questo sovranismo politico antieuropeista al cospetto dell'internazionalismo mercantile della globalizzazione, in una mostruosa fusione di regressione identitaria e dissoluzione dei modelli comunitari; allo stesso modo in cui la Russia di Putin o gli Stati Uniti di Trump alimentano le tradizioni del folklore nazionale vero o presunto. 
Non c'è un solo motivo in nome del quale ci si debba piegare a questo processo, mentre molte buone ragioni ci spingono a criticarlo e contrastarlo, anche servendoci, dal momento che questo è il nostro spazio di intervento, del ragionamento sulla letteratura, sulla sua circolazione nelle istituzioni e al di fuori di esse, e della stessa ridefinizione del canone.

Nel compiere in particolare un ragionamento su quest'ultimo tema, dovremo fare buon uso delle nuove sollecitazioni che da alcuni decenni hanno colpito il campo degli studi letterari; giunte, come ho detto, dai processi di globalizzazione, certo, ma anche da fenomeni politici e culturali fertili e promettenti, come la faticosa e contraddittoria ma pur sempre decisiva costruzione di uno spazio europeo: spazio politico, come sappiamo, e soprattutto economico-finanziario, spazio dunque mercantile, ma anche spazio culturale e identitario. Spazio tuttavia di una possibile e augurabile identità molteplice e inclusiva: l'unica accettabile e praticabile oggi. E in questa prospettiva europea il paesaggio delle letterature nazionali appare costretto a riorganizzarsi in modo significativo, subendo il ridimensionamento dei suoi territori piuttosto eruditi che vivi, e d'altra parte incontrando infine l'opportunità di riconoscere esperienze e linee trascurate quando non sacrificate e perfino esplicitamente conculcate in nome degli stati-nazione e delle loro, troppo spesso violente, dinamiche. E così qualcosa potremo recuperare o valorizzare dalle lingue periferiche della variegata storia italiana, all'interno della quale autori come il romano Belli e il milanese Porta costituiscono punti letterariamente assai alti; e molto avremo occasione di ricevere da un'area come quella iberica, affetta da processi di sovranismo coloniale protratti per secoli, ora a trazione castigliana ora perfino sotto le ambizioni nazionaliste lusitane. Potrà così verificarsi il gradevole paradosso storico di un riconoscimento europeo di alcuni autori di lingua catalana che nello stato-nazione spagnolo faticano a trovare spazio e visibilità adeguati; e potranno circolare liberi nei maggiori paesi d'Europa autori che, come oggi alcuni politici in esilio, sono invece condannati alla reclusione dentro i confini dello stato spagnolo.

Non mi sfugge il tratto utopico di questa speranza. Ma dobbiamo pur rivolgere verso il futuro un progetto, e una opportunità, che ci assegnino oggi un compito e una prospettiva. E credo che sia nostro compito oggi lavorare a questa speranza, per proteggere la possibilità di un nuovo paesaggio e non limitarci a essere i funzionari di una tradizione tanto discutibile e logora. Fra l'altro è questa, per ciò che riguarda gli studi letterari, la tradizione migliore della stessa comparatistica, un continente che appare scosso da turbolenze e ripensamenti, e dal quale potrebbero sorgere nuovi modi di spaziare fra lingue e culture diverse, anche in questo caso superando la prospettiva delle letterature stricto sensu nazionali.

Fra le opportunità di questi ultimi decenni, vanno indubbiamente contati anche gli scoppiettanti processi di rinnovamento teorico-metodologico, con la messa a fuoco dei meccanismi che presiedono alla formazione e alla cristallizzazione del canone letterario. I Cultural Studies hanno operato una critica dell'ideologia, in questo senso, indubbiamente utile e necessaria, benché inciampando spesso nei limiti di alcuni presupposti teorici e politici che li hanno guidati, con una riduzione, per dirla in 
una battuta, della dialettica conflittuale fra barbarie e civiltà insita in ogni processo culturale su cui ha ragionato la Scuola di Francoforte alla condanna univoca dei modelli dominanti. Non si trattava d'altra parte di espellere Shakespeare o Dante dal centro del canone occidentale in nome dell'uso che può esserne stato fatto, quanto di ridefinire il rapporto tra quel centro e ciò che gli è stato attribuito come periferia o, di più, ciò che è stato espunto non solo dal diritto di riconoscimento e, più ancora, dalla opportunità di esistenza. I Postcolonial Studies e i Gender Studies, in particolare, hanno solo eccezionalmente insistito sulla costitutiva problematicità della dinamica e dei valori che accompagnano la formazione del canone, accontentandosi troppo spesso di rivendicare un riconoscimento sostitutivo: un processo che merita indubbiamente di essere compiuto e che si sta finalmente compiendo, ma anche un processo monco ove non venga adeguatamente rivolta l'attenzione al momento generativo dei prodotti di arte e di pensiero, riservata ai maschi bianchi ricchi più ancora di quanto ai loro prodotti sia favorito l'accesso al canone. Infine la contraddizione non è fra Dante e Shakespeare, da una parte, e una periferia più o meno rimossa dall'altra, ma già all'interno stesso di Dante e di Shakespeare, come, e a maggior ragione, all'interno del loro uso nei secoli e oggi. La critica dell'ideologia, ce lo ha insegnato Marx, non deve mirare alla cancellazione del sapere dei ceti dominanti, ma alla sua liberazione.

Abbiamo dunque grandi opportunità per ripensare questo assetto, e in particolare per pensarlo sulla scena europea, di nuovo vitale dopo l'assestamento ottocentesco. E se dalle proposte, e dalle provocazioni, dei Cultural Studies provengono opportunità indiscutibili, benché da trattare con prudenza, sappiamo che cosa non vogliamo fare: difendere il paesaggio della tradizione, e così rischiare di farne un paesaggio mummificato e inerte; come è accaduto ad Harold Bloom, il cui Canone occidentale ha ben mostrato i limiti di un arroccamento conservatore. Come è stato osservato, la pretesa soggettività universalistica della sua proposta naufraga sui limiti delle competenze dello studioso, con il conseguente anglocentrismo. Ben scarso vi è lo spazio riconosciuto alle letterature che oggi possiamo azzardarci a definire iberiche, inclusa la castigliana, e del tutto assente la catalana.

Bloom attribuisce al risentimento -e dunque a una dinamica sbagliata prodotta dai meccanismi dell'esclusione- le critiche mosse al canone occidentale, mentre non individua risentimento nel proprio arroccarsi di fronte alle loro spinte -il risentimento del privilegio assediato. Possiamo sentire la responsabilità di respingere i tratti ingenui o inadeguati o parziali di quegli assalti, ma di certo ci tocca di riconoscere, a differenza di Bloom, il valore e la fondatezza delle loro rivendicazioni e più ancora delle premesse che le muovono.

Dobbiamo dunque rifiutare la modalità archeologico-conservativa, quando non francamente reazionaria, di questo arroccamento; e possiamo ragionare sul canone nell'unico modo teoricamente pertinente, guardandolo a partire dai bisogni del presente, dai nostri nuovi bisogni, a condizione che l'aggettivo possessivo non corrisponda soltanto alle esperienze e alla concezione di un lettore singolo, e possibilmente neppure di un ceto o di un ruolo accademico, a condizione cioè che i bisogni del presente siano vasti quanto le sue potenzialità. E possiamo ragionare dunque sulla permeabilità 
del canone che ci è stato trasmesso, sulla nostra capacità di renderlo tale anche al di là del modello con il quale ha lungamente coinciso lo spazio della letteratura nelle costruzioni identitarie nazionali; così da problematizzarlo, arricchirlo, rinnovarlo, in modo che divenga di nuovo adeguato, o forse per la prima volta adeguato, a una dimensione internazionale e a un progetto universalistico.

Perché questo progetto si svolga, mi pare decisivo insistere sulla categoria di permeabilità: nelle due direzioni, dell'inclusione e dell'esclusione. Dovremo rinunciare, ragionando in un'ottica non specialistica, ai «cavallucci a dondolo» delle nostre identità nazionali e individuali; dovremo tentare un dialogo non spaventato tra il nostro imprinting linguistico ed estetico e un nuovo cosmopolitismo mobile, ben sapendo che ogni ricostruzione di questo intero dell'umanità nel tempo sarà una parte dell'intero cui stiamo aspirando, sarà una sineddoche, e che in questa consapevolezza posa oggi la credibilità della nostra ricerca: la parzialità dovrà sapere di essere tale, senza proporsi più come l'equivalente universalistico della condizione umana. Potremo formare insiemi magari disomogenei e provvisori, ma comunque inclusivi e -lo ripeto- permeabili; e il progetto globalizzato delle economie mondiali sarà affiancato da un progetto cosmopolita dei lettori e degli studiosi di letteratura.

Perché sia davvero permeabile, questo canone cosmopolita dovrà essere inclusivo e aperto, capace di affiancare l'amore per Dante o per Shakespeare alla scoperta incantata dei racconti di Alice Munro e delle atmosfere sospese di Haruki Murakami, dei rivelatori giochi linguistici di Wisława Szymborska e dell'orrore che permea i capolavori di Toni Morrison. In questo modo, fra l'altro, quei centri della presunta identità canonica occidentale, Dante e Shakespeare appunto, smetteranno di essere, come il Colosseo a Roma, inavvertiti spartitraffico di identità sempre più morte e potranno tornare a essere vivi e attuali.

Potremo, anche, guardare per la prima volta, o per la prima volta con adeguata consapevolezza e rispetto, verso letterature emarginate e minorizzate come quella, splendida, in lingua catalana. Ecco, sappiamo quali faticose vicende storiche ne abbiano segnato la marginalizzazione, fino alla persecuzione franchista. Ma proporrei di abbandonare questo punto di vista -che rischia di bloccarci in un gesto di rivendicazione ancora subalterna- a favore di una prospettiva che, se davvero vorrà essere inclusiva e accogliente, dovrà assumersi la responsabilità di essere capace di collocarsi nel presente. Questo non vuol dire che le ragioni di ogni esclusione e di ogni marginalizzazione debbano essere dimenticate, ma che non su di esse potrà poggiare il nuovo cosmopolitismo, quanto meno europeo, né la ridefinizione di contesti e spazi innovativi, come quelli cui allude la decisiva, anche ai nostri fini, categoria di iberismo.

Non si tratta più, o non si tratta soltanto, cioè, di rivendicare la libertà da egemonie disastrose; ma di essere liberi. Il fatto che la letteratura conti oggi meno, molto meno, all'interno dei grandi processi di organizzazione del consenso e di disposizione delle forze del dominio, può permettere a questo territorio di divenire il luogo di un esperimento, o magari di una prefigurazione. Dobbiamo tentare, e possiamo sperare che questo esperimento sia, oltre che il nostro personale contributo a una prospettiva inclusiva e all'universale umano, una possibilità di globalizzazione non fondata sul 
conflitto e sulla cancellazione delle differenze ma sull'arricchimento e sulla inclusione. Un canone più ampio potrà essere la promessa figurale di un mondo più largo, nel quale ci sia posto non solo per gli eredi di Shakespeare capaci di riconoscere in quella grandezza il proprio diritto al privilegio e al dominio, ma anche per altri e per altre, che quel dominio hanno storicamente subito e da quel privilegio sono stati esclusi.

Di questo rinnovamento fa parte il nostro dovere oggi di raccontare storie nuove: parziali, certo, ma comunque meno parziali di quelle che abbiamo ascoltato, o quanto meno, a differenza di quelle e della loro rovinosa pretesa di completezza e di universalità, consapevoli della propria parzialità, consapevoli di essere appunto una sineddoche e una figura.

Nella nuova Europa della generazione, o delle generazioni, Erasmus, in questa Europa che vogliamo cosmopolita, ci aspetta il racconto di una letteratura meno parziale, meno sottomessa al principio egemonico degli stati-nazione, una letteratura che ci permetta di recuperare, soprattutto, le voci forti ed emozionanti di autori che il fiume del processo storico ha lasciato in un'ansa laterale, dove piccole comunità hanno potuto continuare a coltivarne e proteggerne la memoria, non senza il rischio di partecipare, con la loro cura identitaria, al processo di esclusione dal canone internazionale.

2. Per quanto io sappia, il caso della letteratura in lingua catalana è da questo punto di vista forse il più significativo e interessante in Europa. E mi chiedo come sia stato possibile che la funzione strategica di un filosofo, scienziato e innovatore di generi letterari come Ramon Llull sia stata relegata, nella formazione delle persone colte del continente, anche medievisti, in un angolo erudito; mi chiedo come il capolavoro di Martorell sia stato abbandonato a vantaggio di testi, magari non letti, o non integralmente, ma certo largamente presenti nella formazione umanistica continentale come il Cantar de mio Cid o la Chanson de Roland. Importantissimi, l'uno e l'altra, da un punto di vista storico e documentario, e decisivi anzi per comprendere l'ideologia che ha mosso gli eserciti cristiani nell'Europa tra Carlo Magno e la Reconquista, ma oggi lontane dalla nostra sensibilità tanto quanto vicine appaiono invece le invenzioni strabilianti di Tirant lo Blanc, del quale è d'altra parte anche difficile trascurare il valore storico-letterario e perfino genealogico per la nascita del modo nuovo di raccontare, il modo del romanzo e della sua mescolanza costitutiva di generi, così come verrà praticata, non senza un riconoscimento del precedente catalano, da Cervantes.

Proviamo a leggere oggi i versi bellicosi del Cid o della Chanson de Roland, e proviamo poi il piacere sorprendente del Tirant, la sua spregiudicatezza nel trattare delle relazioni tra i generi, nel riconoscere anche alle donne quel diritto al piacere e alla vita che in Italia costituisce uno dei tratti caratterizzanti del capolavoro di Boccaccio. Ecco, non è forse un bel paesaggio quello che disegna questo nuovo immaginario nell'Europa delle corti fra Medioevo e primo Rinascimento, tra la emancipata donna di Bath dei Canterbury Tales, le donne astute o appassionate del Decameron e la complicità di Carmesina e Plaerdemavida nel capolavoro di Martorell? 
Se il canone, come si è più volte giustamente ripetuto, non risponde a una verità, qualunque verità, degli oggetti che colloca e valorizza ma ai bisogni, alle scelte, e in ultima analisi ai valori, in ogni senso, dei soggetti che lo maneggiano, preferiamo che l'Europa di oggi si riconosca nei codici cavallereschi bellicosi e colonialisti del Cid e di Roland, o nell'etica relativa e problematica di Carmesina, nella spregiudicata denuncia dell'Indulgenziere di Chaucer, nella religiosità inclusiva della novella boccacciana dei tre anelli? E non pensiamo anche che la qualità artistica, formale, il piacere della lettura, oggi, sia a sua volta tutto dalla parte di questi tre capolavori, e tanto tanto discosto dall'ideologia raffigurata nei due poemi nazionali di conquista?

Il disincanto dell'Indulgenziere, la perorazione di Ghismunda, l'ironia anticavalleresca di Tirant non sono forse oggi mirabili esperienze di conoscenza formale della nostra vita e della nostra storia, oltre che modelli e possibilità di decostruzione sapiente di tutto ciò che abbiamo smesso di accettare e poi tuttavia visto riproporsi, ancora una volta, terribilmente, nell'Italia fascista, nella Germania di Hitler, nella Spagna di Franco? E che oggi ci perseguita ancora per voce dei sovranismi e del loro sospetto verso ogni forma di alterità, del loro razzismo implicito quando non apertamente professato?

La casa editrice Einaudi ha pubblicato pochi anni fa una grande edizione del Tirant in una collana prestigiosa, i Millenni, benché collocata sul lato del museo e della strenna natalizia, anche per il costo elevato, e i lettori italiani hanno finalmente avuto accesso a questo capolavoro. Quanto tempo e quale lavoro saranno necessari perché esso si veda riconosciuti gli stessi diritti dei «fratelli» europei che ho appena nominato, nel disegnare una costellazione triangolare, che unisca la Valenza del Segle d'Oralla Londra premoderna alla Firenze meridionalizzata da Boccaccio (che si era formato in una Napoli dove la presenza catalana avrebbe rappresentato un momento profondo e significativo di incontro culturale) non possiamo saperlo; ma sappiamo che tra le nostre responsabilità c'è da lavorare a questo riconoscimento, alla logica inclusiva e permeabile che dovrà guidarci, animati anche dal coraggio di denunciare $i$ limiti, storici e attuali, di altri presunti capolavori: destinati a restare nel repertorio dei documenti storici fondamentali, certo, e perfino in quello dei miti fondativi di identità e di Stati, ma non più accolti, come monumenti impropri, nel territorio del canone letterario: quello di cui parliamo ai ragazzi e alle ragazze, e che vorremmo formasse la base della loro personalità di cittadini e del loro sistema di valori.

Gli studiosi di letteratura medievale continueranno a frequentare il poema del Cid e la Chanson de Roland, troveranno nel Nibelungenlied e nel Beowoolf solide ragioni di interesse documentario e fascino nutritivo, e sulla tradizione secolare che si è ispirata a quei modelli e li ha tramandati potranno svolgere una critica dell'ideologia che si configurerà come conoscenza nuova, e più vera, di quelle vicende, e dell'Europa; ma ai nostri allievi, agli studenti delle scuole, ai giovani delle generazioni Erasmus che si incontrano nelle università europee, noi speriamo di poter consegnare la lungimirante critica di quei modelli bellicosi contenuta nei capolavori di Boccaccio, Chaucer e Martorell. 
Un caso non meno clamoroso è quello di Ausiàs March, che appare oggi ai miei occhi quale il lirico d'amore, e non solo d'amore, più grande e vivo dopo Petrarca e prima della fioritura rinascimentale e barocca. Non mi sembra di chiedere troppo a questa nuova Europa che vorremmo contribuire a disegnare secondo parametri cosmopoliti, aspettandomi che faccia posto all'eccezione clamorosa che March porta dentro la dominante petrarchesca, e petrarchista, della lirica d'amore colta; che colga l'attualità, cioè la capacità presente di emozionare e coinvolgere, e far pensare, del suo modo di trattare l'esperienza dell'amore: con le sue contraddizioni, con le sue radicali disarmonie. E non credo sia chiedere troppo, aspettandomi che venga meglio riconosciuto lo slancio realistico di questa esperienza, che mette a disposizione in modo onesto il proprio vissuto, gettandolo tragicamente nell'agone della teologia medioevale.

Sappiamo che a noi spetta oggi il compito, dovuto alla storia e alla verità dei suoi processi, di riconoscere i movimenti di questa confidenza concettuale, di questa partecipazione capillare a un sistema di pensiero e a un immaginario culturale. Ma non di meno sappiamo che a noi spetta il diritto, al cospetto dei lettori vivi che chiediamo per i testi che amiamo, di additare il conflitto tra il realismo psicologico di quella esperienza lealmente rappresentata e il sistema culturale e ideologico con il quale tenta di essere interpretata e redenta. Questa seconda parte del nostro compito non è meno storicamente fondata della prima, se storia è il nostro presente, il nostro sguardo, e non solo il contesto, non immobile d'altra parte neppur esso, nel quale si è svolta la ricerca di March.

Se Petrarca è il grande sperimentatore dell'innamoramento, della rinuncia, della perdita, della sublimazione, e del rimpianto, perfino, nella parte in morte di Laura, di un passato non vissuto, March è quello della carnalità, e del conflitto tra sentire e pensare. Ciò che in Europa abbiamo chiamato «amore», e oggi consideriamo ascrivibile a questo nome, ha bisogno di entrambi, e ha bisogno di esperienze nuove e inclusive, anche di fronte al canone letterario. Costretti a leggere Petrarca, e qualche volta non aiutati a cogliere la complessità del mondo che lo caratterizza, gli studenti italiani hanno smesso o stanno smettendo, da qualche decennio, di cercare nella letteratura risposte ulteriori o alternative, appassionandosi magari ai maledetti francesi e a Baudelaire. Possiamo recuperare la loro attenzione, se il campo di ciò che chiamiamo amore sarà capace di includere $\mathrm{e}$ accogliere anche la radicalità dell'erotismo marchiano? È un esperimento che vale la pena di tentare.

Facendo così disastrosamente a meno dei capolavori scritti in catalano, la cultura europea si è privata, come è possibile vedere anche solo da questi pochi e rapidi esempi, di non poco! E, volendo restare prudentemente nei limiti delle mie competenze ed esperienze di recente iniziato, vorrei concludere con qualche riferimento alla stagione che in Europa, e anche da poco in Italia, abbiamo preso l'abitudine di chiamare modernista, non mancando tuttavia di avvertire che il termine ha nel mondo anglosassone, e oggi italiano, un significato alquanto diverso da quello da tempo praticato in ambito iberico. E che per la letteratura in lingua catalana si potrà ragionare sulla almeno parziale sovrapposizione con il movimento noucentista. 
In catalano hanno scritto non pochi grandi poeti nei primi decenni del Novecento, alcuni dei quali hanno avuto la forza, o la necessità, da esiliati, di varcare i confini nazionali. Penso a J. V. Foix, e a Josep Carner, che mi piacerebbe un giorno tradurre, se mai divenissi capace di parlare del dolore e della perdita con la stessa leggerezza malinconica che lo caratterizza. Ma soprattutto penso a una delle più grandi narratrici del Novecento in Europa, non senza dolermi dell'assenza di un sostantivo che possa includere da solo, davvero, l'universale umano, per dire fra le più grandi e anche fra i più grandi: Mercè Rodoreda.

Il suo realismo modernista la avvicina a Virginia Woolf: una capacità di moltiplicare le prospettive narrative, legandole con una tecnica di montaggio nuova e inedita, non gerarchica e tuttavia non arresa di fronte alla semplice giustapposizione delle esperienze e delle condizioni, una dimensione pluriprospettica che è una linea profonda e sicura come quella tracciata da Lily Briscoe nella conclusione di To the Lighthouse per dare senso al suo quadro, a redimere dal dissolvimento prodotto dalle sole particolarità individuali non redente. E come le narrazioni di Woolf si collocano nell'orizzonte dell'alta società borghese, così quelle di Rodoreda praticano un innovativo realismo dal basso, narrando da una prospettiva nuova e raso terra la vita di quelle che Manzoni chiama «genti meccaniche, e di piccol affare»; liberandone la parzialità da ogni sospetto di populismo paternalista, e piuttosto cercando nei loro movimenti di vita, nelle loro emozioni e sentimenti, una nuova idea di universalità della condizione umana.

Il diritto alla vita interiore dei dominati, che per noi italiani è la grande scoperta di Verga più ancora che di Manzoni, fa di Rodoreda una necessità delle democrazie europee: ci mette nella condizione di praticare storici privilegi di classe, primo fra tutti quello di riconoscerci capaci di emozioni alte e complesse, senza la colpa dell'esclusività; ci rende liberi e uguali al cospetto della condizione umana. $\mathrm{E}$ anche per questo, oltre che per gli indiscutibili valori letterari, la narrativa di Mercè Rodoreda ci appare una delle scoperte più necessarie per l'Europa di questi anni, che d'altra parte la sta offrendo ai suoi lettori, anche in Italia, con molte traduzioni.

Come quella di alcuni suoi personaggi indimenticabili, le voci letterarie che parlano in catalano sembrano oggi raccontare, emblematicamente, la storia possibile di una nuova Europa, sembrano incarnare -dal margine, dalla periferia- la possibilità di un nuovo centro: plurale, accogliente, inclusivo; permeabile. Noi aspettiamo questa Europa e siamo già fiduciosi di farne parte. 